\title{
Role of Magnetic Resonance Imaging in Evaluation of Acute Pancreatitis
}

\author{
SHAIMAA H. BAKR, M.Sc.*; HASSAN I. MEGALLY, M.D.*; REEM M. EL-KADY, M.D.**** and \\ WAEL A. ABBAS, M.D.*** \\ The Department of Diagnostic Radiology*, Faculty of Medicine, Assiut University, Assiut, Egypt, \\ The Department of Radiology and Medical Imaging**, College of Medicine, Taibah University, Madinah, Saudi Arabia and \\ The Department of Internal Medicine***, Faculty of Medicine, Assiut University, Assiut, Egypt
}

\begin{abstract}
Background: To investigate the role of dynamic Magnetic Resonance Imaging (MRI) in diagnosing and assessing the severity of Acute Pancreatitis (AP) patients and role out pancreatic necrosis.

Aim of Study: The aim of the study was to determine the effectiveness of dynamic MRI in the diagnosis of AP and assessing the severity of AP by detection and delineation of extension of pancreatic necrosis in comparison to clinical scoring systems.
\end{abstract}

Material and Methods: Thirty four patients with clinical diagnosis of acute pancreatitis underwent magnetic resonance imaging using $1.5 \mathrm{~T}$ machine include $\mathrm{T} 1, \mathrm{~T} 2$ weighted imaging, SPAIR and dynamic contrast. Magnetic Resonance Severity Index (MRSI) was used to assess the severity and correlated with clinical scoring based on revised Atlanta classification and Acute Physiology and Chronic Health Evaluation II (APACHE II) score.

Results: T2-SPAIR is the best sequence to diagnose AP. The MRSI significantly correlates with both revised Atlanta and APACHE II scores in differentiating mild cases of AP from moderate edematous and severe necrotizing cases ( $p$ $<0.001$ for revised Atlanta and 0.004 for APACHE II.

Conclusion: Dynamic MRI help to diagnose mild forms of AP and differentiate it from other causes of abdominal pain.MRI can be used in combination with clinical scoring for diagnosis of AP and accurate assessment of disease severity.

Key Words: Acute pancreatitis - MRI-Revised Atlanta APACHE II score.

\section{Introduction}

ACUTE Pancreatitis (AP) is one of the most commonly observed pancreatic disorders [1].

$\mathrm{AP}$ is the most terrible of all the calamities that occur in connection with the abdominal viscera. The suddenness of its onset, the illimitable agony

Correspondence to: Dr. Shimaa H. Bakr,

E-Mail: drshemo1989@vahoo.com which accompanies it, and the mortality attendant all render it most formidable of catastrophes [2] .

The incidence of $\mathrm{AP}$ has been on the rise worldwide [3]. AP has a yearly incidence of approximately $13-45$ new cases per 100,000 per year [4]

AP is a potentially fatal disease which has to be accurately diagnosed with a mortality rate of over $10 \%$ [5]

Necrotizing disease and advanced age are associated with increased mortality risk. Early diagnosis is essential for effective and timely treatment of disease and avoidance of complications [6].

AP is an inflammatory condition caused by intracellular activation and extravasation of inappropriate proteolytic enzymes, leading to autodigestion of the gland, determining destruction of pancreatic parenchyma and peripancreatic tissues [7].

The most prevalent etiologic factors in AP are choledocholithiasis and alcoholism [6] .

Less common causes include hypertriglyceridemia, hypercalcemia, drugs, and autoimmune diseases [7].

Imaging plays a vital role in the management of pancreatitis. It enables diagnosis and differentiation of the severity of this condition. This is crucial for guiding clinical management and has prognostic value. In addition, it helps to identify and manage the associated complications with image-guided drainage and aspiration [8]

Magnetic Resonance Imaging (MRI) is becoming increasingly important and is often the preferred imaging modality to evaluate a variety of pancreatic disease processes [9] 


\section{Patients and Methods}

This is a prospective study that was carried out in the Radiology Department of Assiut University Hospital during the period from April 2015 to May 2016.

This study was performed on 34 patients referred to us from General Surgery and Internal Medicine Departments diagnosed as acute pancreatitis based on clinical diagnostic guidelines. $\mathrm{Ab}$ dominal contrast enhanced MRI was done for all patients.

\section{Inclusion criteria:}

Patients in different ages who have two of the following three features to make a diagnosis of AP: Characteristic abdominal pain, an elevated serum amylase or lipase > 3 times the upper limit of normal, and characteristic findings of AP on ultrasound or CECT scan.

\section{Exclusion criteria:}

Patients with:

- History of chronic pancreatitis;

- History of pancreatic surgery;

- Any general contraindication of MRI as presence of any paramagnetic substance as pacemakers or those with claustrophobia; and

- Severely ill patients or inability of patients to cooperate when performed MR imaging.

\section{Each patient was subjected to the following:}

- Clinical and laboratory assessment:Performed by the referring physician;

a- Clinical history and physical examination: Including the vital signs.

b- Laboratory investigation including: Arterial $\mathrm{PH}$, serum sodium, potassium, creatinine, hematocrite, white blood count, $\mathrm{O}_{2}$ saturation.

c- Clinical scoring:

1- Revised Atlanta classification: Patients were categorized into mild, moderate and severe acute pancreatitis based on the presence or absence of local and systemic complications and organ failure.

- Organ failure is defined by the modified Marshall scoring system.

- Local complications include necrosis (detected by CECT imaging which was done for all patients), fluid collections, gastric outlet dysfunction, splenic and portal vein thrombosis, and colonic necrosis.
- Systemic complication is defined as exacerbation of pre-existing co-morbidity, such as coronary artery disease or chronic lung disease, precipitated by the AP.

2- Calculation of the APA CHE II scores: Within the first 24 hours of admission based on axillary temperature, mean arterial blood pressure, heart rate, respiratory rate, oxygenation, arterial $\mathrm{PH}$, serum sodium, potassium, creatinine, hematocrite, white blood count and Glasgow coma scale. All values were taken from a score from 0 to 4 points, with 0 points being normal and 4 points being the most abnormal. The sum of the points constituted the acute physiologic score. A point weighting for patient age and a point weighting for chronic health problems are added to acute physiologic score and constitute the APACHE II score.

- Radiological assessment: MRI abdomen Dynamic contrast:

The standard MRI protocol performed for all patients was as follows:

MRI examination was performed using a 1.5 Tesla MR Imager scanner (Achieva; Philips Medical Systems) using abdominal phased array Torso coil in the supine position with small field of view and thin sections. The imaging to all patients was done one week after the onset of the attack.

The following sequences were obtained:

- Coronal turbo spin-echo T2-weighted image: Repetition time (TR) 526ms, Echo time (TE) $80 \mathrm{~ms}$, slice thickness $5 \mathrm{~mm}$, Field of View (FOV) 425mm, Matrix 304 X 264, Flip Angle (FA) (900) and Acquisition time 1:06min.

- Axial turbo spin-echo T2- HR weighted image: TR $1670 \mathrm{~ms}$, TE $100 \mathrm{~ms}$, Slice thickness 5mm, FOV 375 mm, Matrix 400X224, FA (900) and Acquisition time 4:03min.

- Axial T1 FFE-weighted image: TR260ms, TE 46ms, slice thickness 5mm, FOV 375mm, Matrix 240 X 118, FA (800) and Acquisition time 15sec.

- Axial T2-SPAIR weighted sequence: TR 768ms, TE $80 \mathrm{~ms}$, slice thickness $7 \mathrm{~mm}$, FOV $273 \mathrm{~mm}$, Matrix 304 X 209, FA (900) and Acquisition time $2: 36 \mathrm{~min}$.

- MRCP was done for 15 cases (to exclude intra or extra hepatic biliary dilatation), sections were obtained in axial and coronal TSE T2-weighted sequence with the use of breathing averaged 3D fast spin-echo technique or thick single slab $2 \mathrm{D}$ single shot fast spin-echo technique.

- Dynamic contrast-enhanced MRI: Axial pre and post contrast fat saturated T1 THRIVE images 
were obtained immediately after manually injected gadolinium chelate at a dose of $0.1 \mathrm{mmol} / \mathrm{kg}$ of body weight; three phases were obtained, arterial, venous/portal and parenchymal. An additional delayed phase was acquired and images were obtained sequentially at $30,70,120 \mathrm{sec}$ and at 4 minute. Slice thickness $1.3 \mathrm{~mm}$, FOV $375 \mathrm{~mm}$, Matrix 180 X 142, FA (100) and Acquisition time $11 \mathrm{sec}$.

\section{Data analysis and image interpretation:}

$M R$ imaging analysis:

MR images were analyzed for the following:

- Enlargement of pancreas; the enlarged pancreas was defined as anterior posterior diameter $\geq 3 \mathrm{~cm}$ on axial images.

- Signal intensity of the pancreas on different sequences: T2WI, T1WI and T2-SPAIR.

- Degree of uptake of contrast medium by pancreatic parenchyma, extent of necrosis of pancreatic parenchyma $(<30,30-50,>50 \%)$.

- Presence of peripancreatic fluid collection.

- Related extra-pancreatic findings as presence of biliary stones, extra hepatic biliary dilation, portal venous thrombosis, splenic vein thrombosis or arterial (pseudo)aneurysm.

- Presence of pleural effusion or ascites.

Interpretation of DCE-MRI:

The severity of acute pancreatitis was analyzed using MRSI criteria shown in (Table 1) which were derived from CTSI criteria and include Balthazar grades (A-E) and the extent of pancreatic necrosis.

Table (1): MRSI scoring system.

\begin{tabular}{lll}
\hline \multicolumn{1}{c}{ Characteristics } & Score Grade \\
\hline Prognostic indicators & & \\
\hline $\begin{array}{l}\text { Pancreatic inflammation: } \\
\text { - Normal pancreas }\end{array}$ & 0 & A \\
- Focal or diffuse enlargement of the pancreas & 1 & B \\
- Intrinsic pancreatic abnormalities with & 2 & C \\
$\quad$ inflammatory changes in the peripancreatic & & \\
fat & & \\
- Single ill defined fluid collection & 3 & D \\
- Two or more poorly defined fluid collections & 4 & E \\
Pancreatic necrosis: & & \\
- No necrosis & 0 & \\
- <30\% & 2 & \\
- 30-50\% & 4 & \\
- >50\% & 6 & \\
\hline
\end{tabular}

MRSI: Balthazar score $+\%$ of necrosis. The maximum score that can be obtained is 10 .
- Mild pancreatitis (interstitial): Balthazar B or C, without pancreatic or extra pancreatic necrosis.

- Moderate pancreatitis (exudative): Balthazar D or E, without pancreatic necrosis; peripancreatic collections are due to extra pancreatic necrosis.

- Severe pancreatitis (necrotizing): With pancreatic necrosis.

Pancreatic necrosis was defined as wellmarginated areas of signal intensity different from the signal intensity of the normal pancreas on non enhanced imaging and the absence of enhancement on enhanced imaging.

\section{Statistical analysis:}

Data were checked, entered and analyzed using computer programs Microsoft Excel 2010 and statistical program for social science, Version 15 (SPSS Inc., Chicago, Illinois, USA) as follows: Description of quantitative variables as range, mean, Standard Deviation (SD), median, frequencies (number of cases) and percentages when appropriate; description of qualitative variables as number and percentage, sensitivity, specificity ,accuracy, predictive values of positivity and negativity of dynamic MRI study were recorded. $p$ values $\leq 0.05$ was considered statistically significant.

\section{Results}

Thirty four patients (10 male and 24 female patients with ages ranging from 7 to 74 years and average age of $41.4 \pm 16.9$ years) were included in our study.

The causes of acute pancreatitis in our study shown in Fig. (1) were as follows: Gall stones in 20 case $(58.8 \%)$, post ERCP in 3 cases $(8.8 \%)$, alcohol in 1 case $(2.9 \%)$, trauma in 1 case $(2.9 \%)$, hypertriglyceridemia in 1 case $(2.9 \%)$; in the remaining 8 cases $(23.5 \%)$, no cause was found (idiopathic).

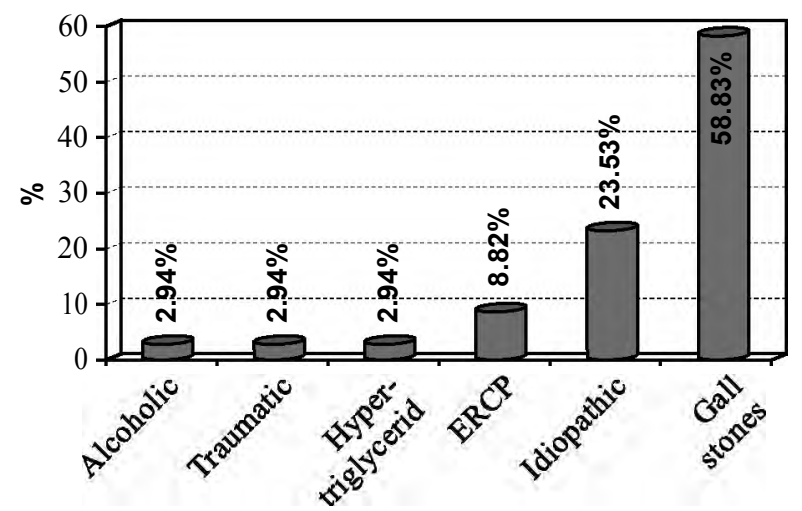

Fig. (1): Diagram showing different underlying causes of AP. 
Severity of AP based on the revised Atlanta classification:

In our study, 20 patients (58.8\%) had moderate AP, 10 patients $(29.4 \%)$ had mild AP and 4 patients $(11.8 \%)$ had severe AP.

Severity of AP based on APACHE II score:

In our study, 20 patients $(58.8 \%)$ scored on APACHE II score less than 8 (mild to moderate) and remaining 14 patients $(41.2 \%)$ scored more than 8 (severe).

\section{MRI findings:}

- Pancreatic size:

In our study, pancreas found to be enlarged in 26 cases $(76.5 \%)$ and of average size in 8 cases $(23.5 \%)$.

\section{- Signal intensity:}

Out of 34 patients, most cases 22 (64.7\%) exhibit no signal change (isointense to the liver) in T1WI, in 7 cases $(20.6 \%)$ the signal was hypointense and in the remaining 5 cases $(14.7 \%)$ the signal was hyperintense.

In T2WI, out of 34 patients, 24 case $(70.6 \%)$ exhibit hyperintense signal (compared to the liver), in the remaining 10 cases $(29.4 \%)$ there was no signal change (isointense). In T2 SPAIR, out of 34 patients, most cases 32 case $(94.1 \%)$ exhibit hyperintense signal (compared to the liver), in the remaining 2 cases $(5.9 \%)$ there was no signal change (isointense) (Table 2).

Table (2): Signal intensities of the pancreas in different sequences.

\begin{tabular}{lcc}
\hline Signal intensity & Frequency & Percentage \\
\hline$T 1$ WI: & & \\
Hypointense & 7 & 20.6 \\
Isointense & 22 & 64.7 \\
Hyperintense & 5 & 14.7 \\
$T 2 W I:$ & & \\
Hyperintense & 24 & 70.6 \\
Isointense & 10 & 29.4 \\
$T 2$-SPAIR: & & \\
Hyperintense & 32 & 94.1 \\
Isointense & 2 & 5.9 \\
\hline
\end{tabular}

- Enhancement after IV contrast administration:

Out of 34 patients in our study, 29 patients $(85.3 \%)$ were diagnosed as interstitial edematous AP and showed delayed homogenous enhancement, while 5 patients $(14.7 \%)$ were diagnosed as necrotizing AP and showed areas of no enhancement at all phases. Among the 5 patients with necrotizing AP, there were 2 patients ( $40 \%)$ with necrosis less than $30 \%, 1$ patient $(20 \%)$ with necrosis between $30 \%$ and $50 \%$, and 2 patients $(40 \%)$ with necrosis more than $50 \%$ of the total pancreatic volume.

- Balthazar MR severity score and index:

Patients were assigned severity index based on the inflammatory changes and the extent of necrosis on MRI according to Balthazar MR severity index.

In our patients, the range of the MRI score was 2-9 with mean \pm SD $3.41 \pm 1.62$ and grades of AP according to MRSI was as follows; 11 patients (32.4\%) graded as mild Fig. (1), 18 patients $(52.9 \%)$ graded as moderate Fig. (2), and 5 patients $(14.7 \%)$ graded as severe (necrotizing) Fig. (3).

- Correlation between severities of AP based on $M R$ severity index versus revised Atlanta classification:

From 11 cases diagnosed as mild AP by MRSI, 10 cases diagnosed as mild, 1 case as moderate and 0 cases as severe using the revised Atlanta classification.

From 18 cases diagnosed as moderate AP by MRSI, 18 cases diagnosed as moderate, 0 cases as mild and 0 cases as severe using the revised Atlanta classification.

From 5 cases diagnosed as severe AP by MRSI, 4 cases diagnosed as severe, 1 case as moderate and 0 cases as mild using the revised Atlanta classification (Table 3).

Table (3): Correlation between MRSI and revised Atlanta classification in predicting the severity of AP.

\begin{tabular}{|c|c|c|c|c|c|c|c|}
\hline \multirow{3}{*}{$\begin{array}{l}\text { MRI } \\
\text { grade }\end{array}$} & \multicolumn{6}{|c|}{ Grade according to revised Atlanta } & \multirow{3}{*}{$\begin{array}{c}p- \\
\text { value }\end{array}$} \\
\hline & \multicolumn{2}{|c|}{ Mild } & \multicolumn{2}{|c|}{ Moderate } & \multicolumn{2}{|c|}{ Severe } & \\
\hline & No. & $\%$ & No. & $\%$ I & Jo. & $\%$ & \\
\hline Mild & 10 & 100.0 & 1 & 5.0 & 0 & 0.0 & $<0.001 * *$ \\
\hline Moderate & 0 & 0.0 & 18 & 90.0 & 0 & 0.0 & \\
\hline Severe & 0 & 0.0 & 1 & 5.0 & 4 & 100.0 & \\
\hline
\end{tabular}

- Correlation between severities of AP based on $M R$ severity index versus APACHE II score:

From 29 cases diagnosed as mild to moderate AP by MRSI, 20 cases diagnosed as mild to moderate and 9 cases as severe using the APACHE II score.

All 5 cases diagnosed as severe AP by MRSI, were diagnosed as severe using the APACHE II score (Table 4). 
Table (4): Correlation between MRSI and APACHE II score in predicting the severity of AP.

\begin{tabular}{|c|c|c|c|c|c|}
\hline \multirow{3}{*}{ MRI grade } & \multicolumn{4}{|c|}{ Grade according to APACHE II } & \multirow{3}{*}{$\begin{array}{c}p- \\
\text { value }\end{array}$} \\
\hline & \multicolumn{2}{|c|}{ Mild to moderate } & \multicolumn{2}{|c|}{ Severe } & \\
\hline & No. & $\%$ & No. & $\%$ & \\
\hline Mild to moderate & 20 & 100 & 9 & 64.3 & $0.004 * *$ \\
\hline Severe & 0 & 0.0 & 5 & 35.7 & \\
\hline
\end{tabular}

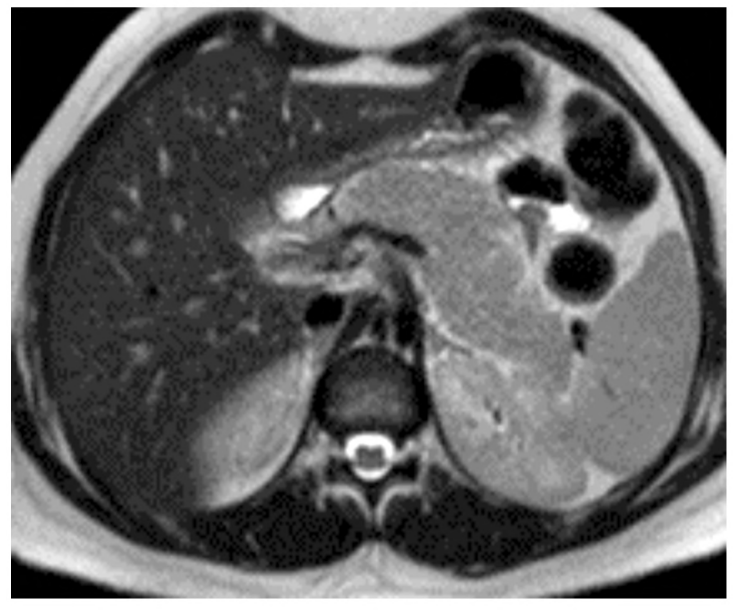

(A) Axial T2WI.

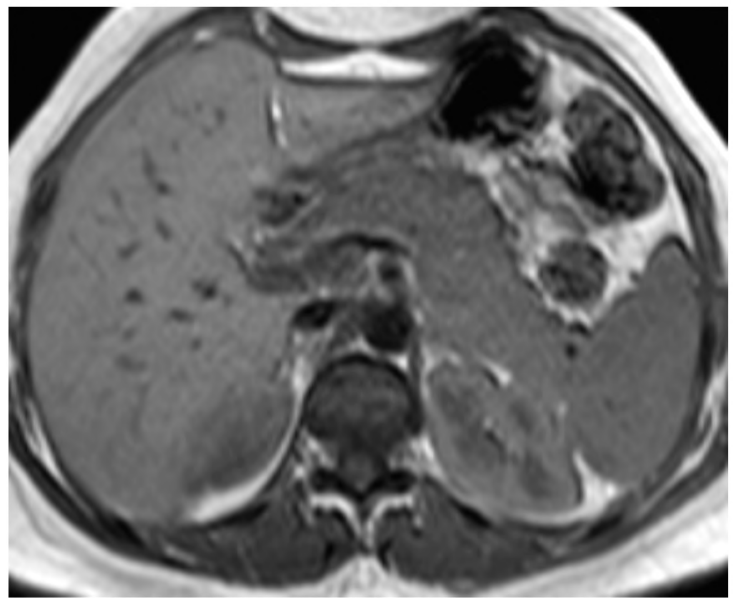

(C) Axial T1WI.

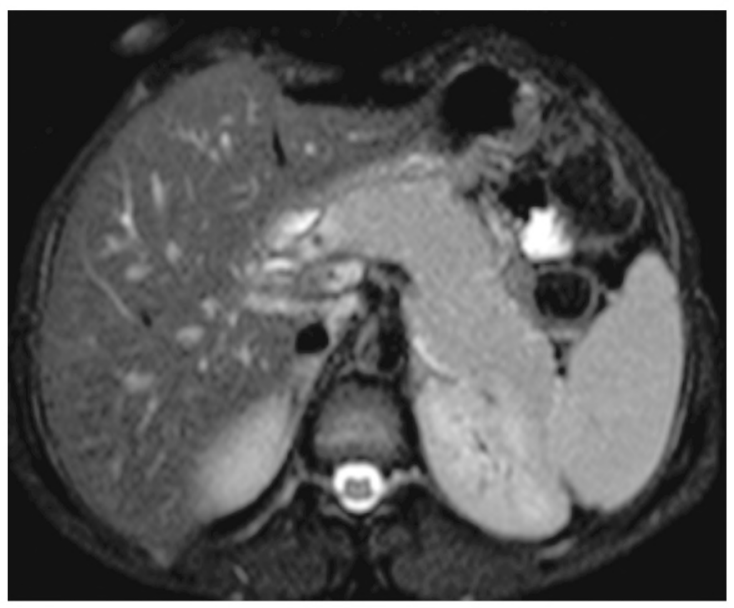

(B) Axial T2-SP AIR.

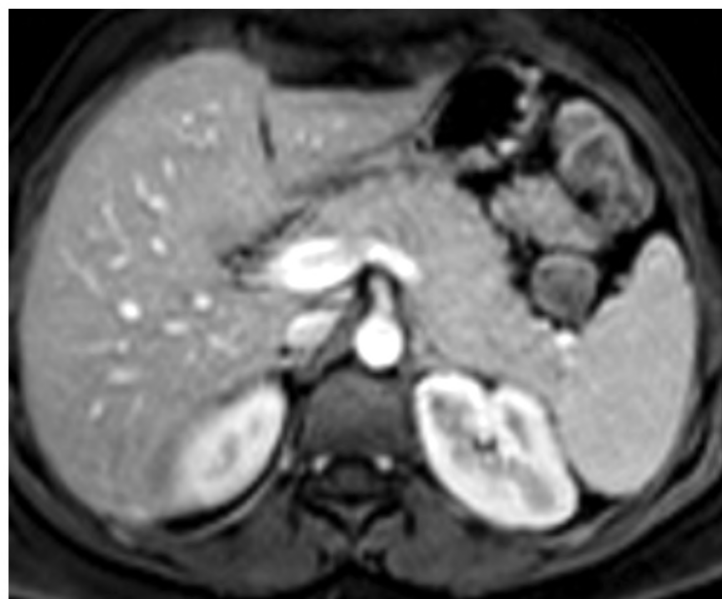

(D) Axial T1 fat sat.post contrast.

Fig. (1): Female patient, 20 years old, presented by diffuse abdominal pain with elevated serum amylase. (A, B, C \& D) Images show diffuse enlargement of the pancreas with signal intensity as follows: In T2WI \& SPAIR (A, B): Diffuse hyperintense signal (relative to the liver). In T1WI (C): Diffuse hypointense signal (relative to the liver). In T1 fat suppression post contrast (D): Delayed homogenous enhancement seen in the portal phase. MRSI: Score: 2 Grade: Mild edematous pancreatitis.

\section{Regarding clinical scoring:}

1- Revised Atlanta classification: Mild pancreatitis.

As patient had no local or systemic complication or organ failure.

2- APACHE II score: 3 so, grade: Mild to moderate. 


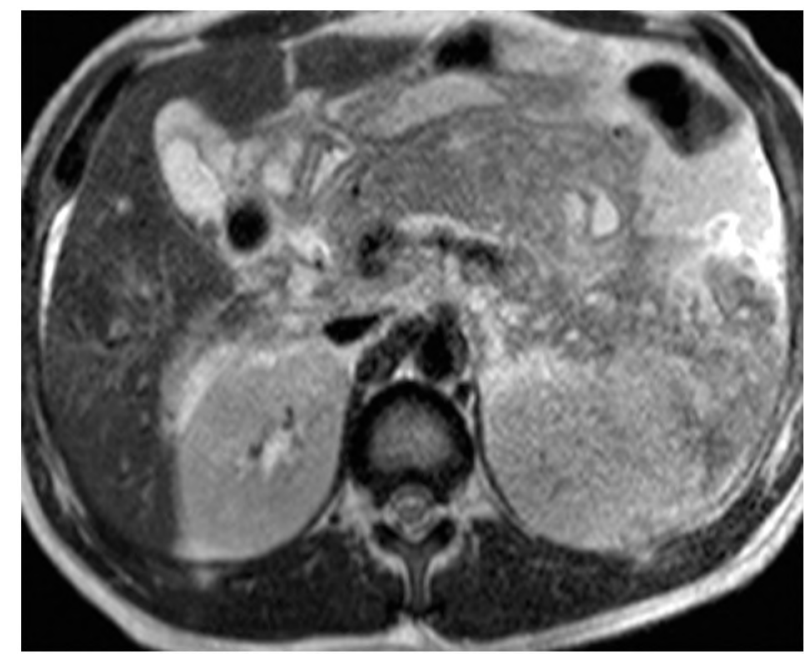

(A) Axial T2WI.

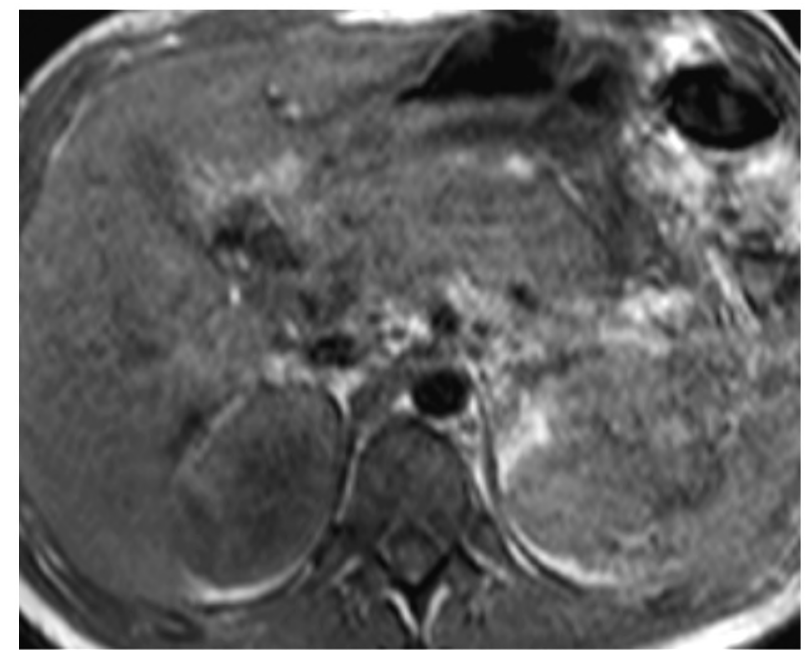

(C) Axial T1WI.

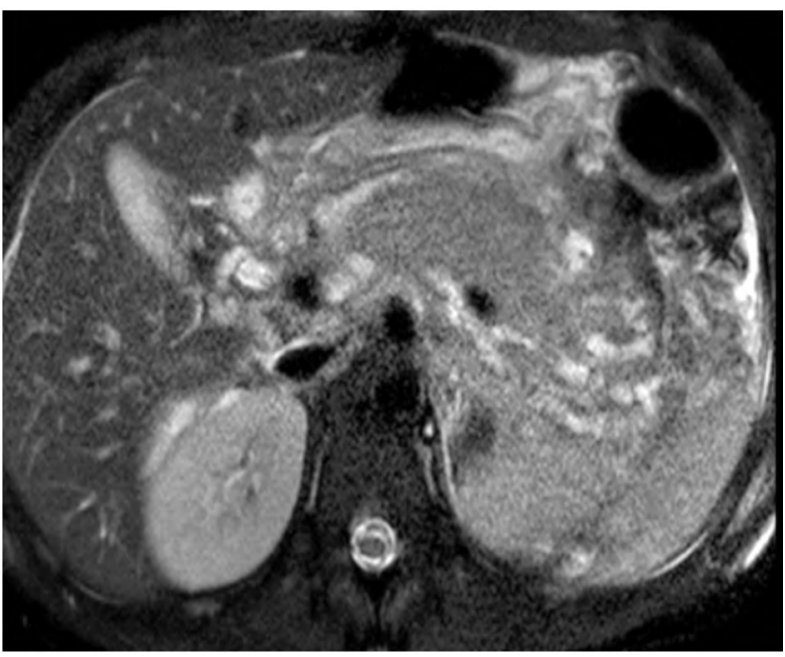

(B) Axial T2-SP AIR.

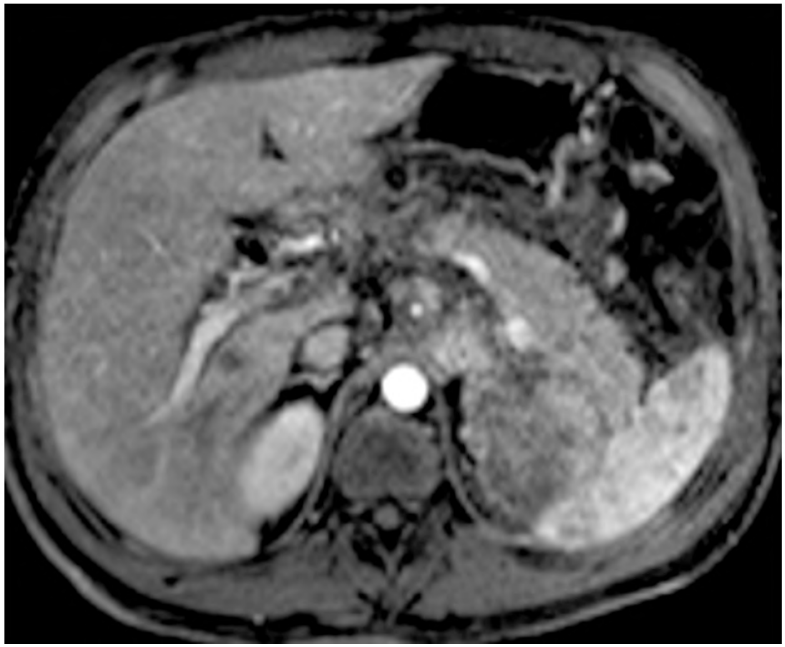

(D) Axial T1 fat sat.post contrast.

Fig. (2): Male patient, 30 years old, presented by severe epigastric abdominal pain with elevated serum amylase after ERCP. (A, B, C \& D) Images showed diffuse enlargement of the pancreas with signal intensity as follows: In T2WI \& SPAIR (A, B): Diffuse hyperintense signal relative to the liver with collection seen peripancreatic \& at the anterior para renal spaces on both sides. In T1WI (C): Isointense to the liver with blurring of pancreatic borders. In T1 fat suppression Post contrast (D): Delayed homogenous enhancement in portal phase. GB shows small signal void stones. MRSI: Score: 4 grade: Moderate edematous pancreatitis.

Regarding clinical scoring:

1- Revised Atlanta classification: Moderate pancreatitis.

As patient had local complication (acute peripancreatic fluid collection).

2- APACHE II score: 4 so, grade: Mild to moderate. 


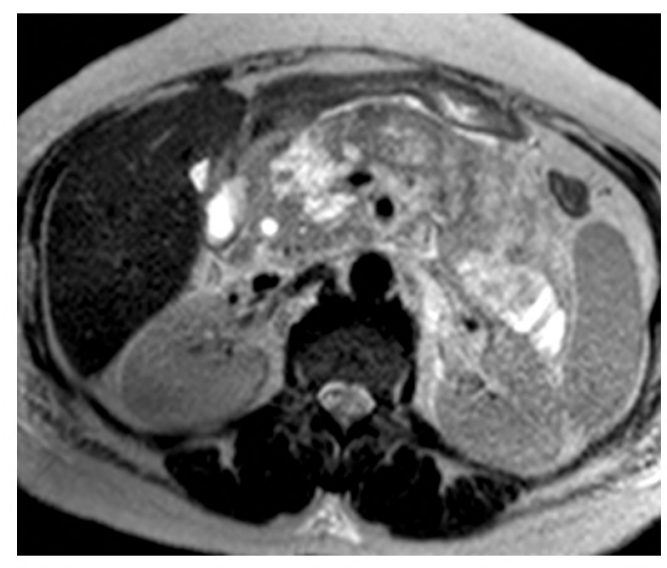

(A) Axial T2WI

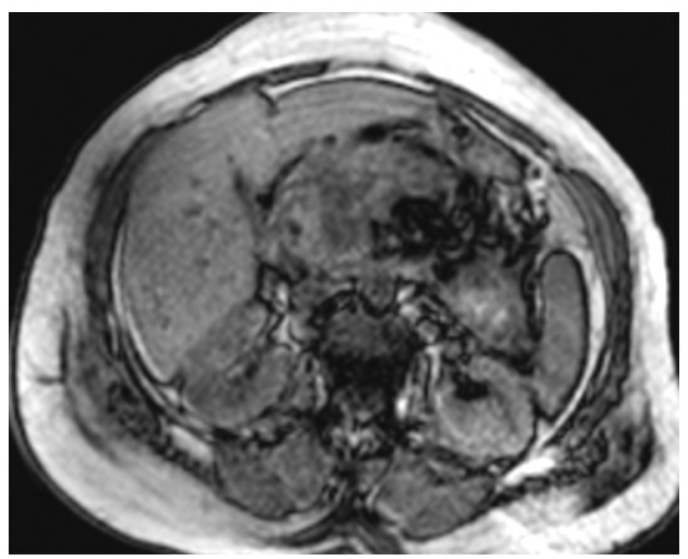

(C) Axial T1WI.

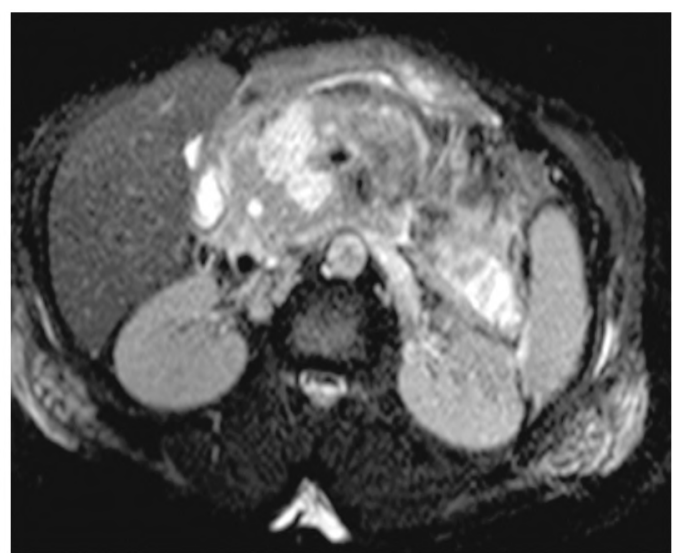

(B) Axial T2-SP AIR.

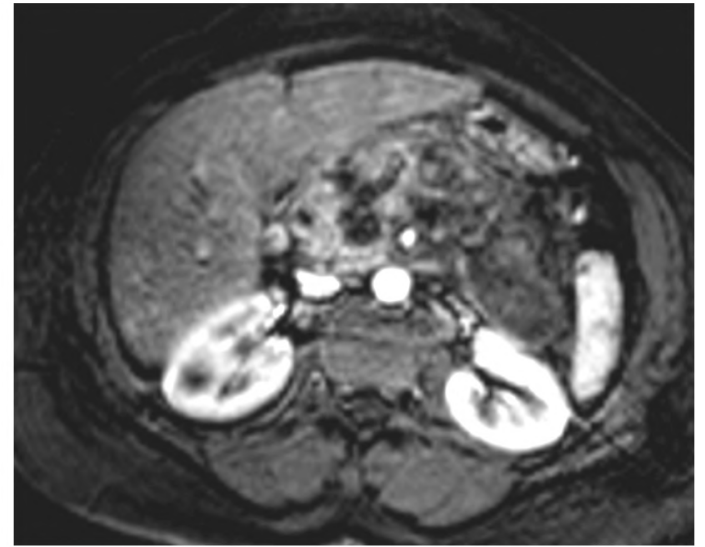

(D) Axial T1 fat sat.post contrast.

Fig. (3): Female patient, 50 years old, presented by severe epigastric pain and vomiting with elevated serum amylase. (A, B, C \& D) Images showed diffuse enlargement of the pancreas with signal intensity as follows: In T2WI \& SPAIR (A, B): Diffuse hyperintense signal with well defined areas of more increase in signal intensity in the body and tail. In T1WI (C): Diffuse hypointense signal with more decrease in the signal in the previously described areas. In T1 post contrast (D): Multiple well defined areas of no enhancement at any phase in the body and tail suggesting necrotic areas. MRSI: Score: 7 grade: Severe necrotizing pancreatitis.

Regarding clinical scoring:

1- Revised Atlanta classification: Severe pancreatitis.

As patient had persistent organ failure (systolic blood pressure was 80 for the first 2 days) and local complication (necrosis by CECT).

2- APACHE II score: 8 so, grade: Severe.

\section{Discussion}

Early diagnosis, accurate staging, and immediate treatment may improve the outcome of AP. However, there is no single method to establish the diagnosis of AP or grade disease severity. Clinical signs such as epigastric pain are non specific and can be absent in up to $10 \%$ of patients with pancreatitis. Although serum lipase and amylase levels have commonly been used to diagnose pancreatitis, they are normal in up to $20 \%$ of cases [1].

Our study included a total of 34 patients with diagnostic criteria of AP who underwent dynamic contrast MRI.
In our study, all patients were subjected to clinical and laboratory assessment. For full evaluation of the pancreatic parenchyma we used axial T2WI, Axial T2-SPAIR, Axial T1WI and Axial T1 fat suppression pre and post contrast. Coronal T2WI was also done for evaluation of the whole abdomen. These sequences were also used by Salik AE \& colleagues [10]. We also used a small field of view in T2-SPAIR sequence to increase the magnification of the pancreas with better image quality and reduce the artifacts.

In our study, we correlated the MRSI with revised Atlanta classification and APACHE II score in predicting the severity of AP. A study done by 
Tang W, et al., studied the correlation between established MRSI in AP and the APACHE II score [11].Another study performed by Salik AE, et al., studied the correlation between MRSI, APACHE II and also evaluated the association between the severity and the clinical outcome in AP [10]

The radiologist who analyzed MR images was blinded to the clinical score of the patients.

In our study, pancreatitis was common in females $(70.6 \%)$ than males $(29.4 \%)$; this is in agreement with the study done by Salik AE \& colleagues [10].

Also, the mean age \pm SD was $41.4 \pm 16.9$, this is similar to study done by Suvarna R \& colleagues [12].

In the present study, the most frequent cause was gall stones $(58.8 \%)$ followed by post ERCP $(8.8 \%)$, this is reported by Salik, et al., and Bollen \& colleagues $[10,13]$ however, this is disagreed with a study done by Park $\mathbf{J} \&$ colleagues, where the alcohol was the most common cause and accounting for $(49.9 \%)$ [14]

On analyzing severity of AP based on revised Atlanta classification, there were $58.8 \%$ moderate AP, $29.4 \%$ mild AP and $11.8 \%$ severe AP, this comes on agreement with a study done by Morappanavar \& colleagues which reported nearly the same prevalence of different grades of severity of AP [15]

On analyzing the MRI findings; the pancreatic parenchymal changes including changes of signal intensity, peripancreatic fat stranding and peripancreatic fluid collection are best depicted on T2WI and on T2-SPAIR with sensitivity of $70.6 \%$ and $94.1 \%$ respectively, 10 out of 11 cases of mild edematous pancreatitis showed change of signal intensity in T2-SPAIR so, it had $91 \%$ sensitivity in detecting mild cases, this is agreed with results of Zhang X-M, et al., and Kim YK, et al., [16,17] Also, the dynamic contrast could detect $100 \%$ of the necrotic pancreatic segments in the necrotizing cases.

In our study, grading of AP according to MRSI, which depends on pancreatic morphological changes and the extent of pancreatic necrosis detected by dynamic contrast, was $29.4 \%$ graded as mild AP, $55.9 \%$ graded as moderate AP and $14.7 \%$ graded as severe (necrotizing) AP, among the 5 patients with necrotizing AP, there were $40 \%$ with necrosis less than $30 \%, 20 \%$ between $30 \%$ and $50 \%$, and $40 \%$ more than $50 \%$ of the total pancre- atic volume, this is similar to the results reported by Li X, et al., and Tang W, et al., where $36.4 \%$ of the patients had mild AP, $50.5 \%$ had moderate AP and $13.6 \%$ had severe AP [11,18]

To our knowledge, there was no previous studies correlate the MRSI with the revised Atlanta classification in the prediction of severity of AP. In our study, we found significant correlation between the MRSI and the revised Atlanta classification for $\operatorname{AP}(p \leq 0.001)$.

In the present study, we found significant correlation between the MRSI and the APACHE II score in predicting mild and moderate grades of AP while the APACHE II score overestimates the severe (necrotizing) form of AP. Same results were mentioned by Lankisch PG \& colleagues who reported that the APACHE II score of eight or more points overestimated the disease in $28 \%$ of patients with edematous AP [19]. This could be explained by the fact that the APACHE II score is based on the clinical status of the patients and mainly reflects the systemic complications, while MRI emphasize on the morphology and extent of pancreatic and peripancreatic tissue alterations. Other study performed by Tang W \& colleagues reported no significant correlation between the MRSI and the APACHE II score [11].

\section{Conclusion:}

Dynamic MRI help to diagnose mild forms of AP and differentiate it from other causes of abdominal pain. MRI can be used in combination with clinical scoring for diagnosis of AP and accurate assessment of disease severity.

\section{Conflicts of interest:}

There are no conflicts of interest.

\section{References}

1- YENCILEK E., TELLI S., TEKESIN K., et al.: The efficacy of diffusion weighted imaging for detection of acute pancreatitis and comparison of subgroups according to Balthazar classification. Turk. J. Gastroenterol., 25 (5): 553-7, 2014.

2- CHOUDHARY S.K., ARYA V.R., KUMAR B., et al.: A comparative study of different predictive severity scoring system for acute pancreatitis in relation to outcome. International Journal of Recent Trends in Science and Technology, 15 (1): 89-93, 2015.

3- KUO D.C., RIDER A., ESTRADA P., et al.: Acute Pancreatitis: What's the Score? The Journal of Emergency Medicine, 48 (6): 762-70, 2015.

4- YADAV D. and LOWENFELS A.: The epidemiology of pancreatitis and pancreatic cancer. Gastroenterology, 144 (6): 1252-61, 2013. 
5- AYDIN H., TATAR I.G. and HEKIMOGLU B.: The Role of Diffusion Weighted MR Imaging in the Diagnosis of Acute Pancreatitis. International Journal of Emergency Mental Health and Human Resilience, 2014.

6- HOCAOGLU E., AKSOY S., AKARSU C., et al.: Evaluation of diffusion-weighted MR imaging in the diagnosis of mild acute pancreatitis. Clinical Imaging, 39 (3): 4637, 2015.

7- CUNHA E.F.D.C., ROCHA M.D.S., PEREIRA F.P., et al.: Walled-off pancreatic necrosis and other current concepts in the radiological assessment of acute pancreatitis. Radiologia Brasileira, 47 (3): 165-75, 2014.

8- KOO B., CHINOGUREYI A. and SHAW A.: Imaging acute pancreatitis. The British Journal of Radiology, 83 (986): 104-12, 2010

9- O'NEILL E., HAMMOND N. and MILLER F.H.: MR imaging of the pancreas. Radiologic clinics of North America, 52 (4): 757-77, 2014.

10- SALIK A.E., ISLIM F., TEKDOS Y., et al.: The diagnostic role of DW-MRI in acute pancreatitis: Assessment of the clinical outcome. European Society of Radiology, Congress ECR 2013.

11- TANG W., ZHANG X.M., XIAO B., et al.: Magnetic resonance imaging versus Acute Physiology And Chronic Healthy Evaluation II score in predicting the severity of acute pancreatitis. European Journal of Radiology, 80 (3): 637-42, 2011.

12- SUVARNA R., PALLIPADY A., BHANDARY N., et al.: The clinical prognostic indicators of acute pancreatitis by APACHE II scoring. Journal of Clinical and Diagnostic Research, 5 (3): 459-63, 2011.

13- BOLLEN T., SINGH V., MAURER R., et al.: A Comparative Evaluation of Radiologic and Clinical Scoring Systems in the Early Prediction of Severity in Acute Pancreatitis. Am. J. Gastroenterol., 107 (4): 612-9, 2012.

14- PARK J., JEON T., HA T., et al.: Bedside index for severity in acute pancreatitis: Comparison with other scoring systems in predicting severity and organ failure. Hepatobiliary \& Pancreatic Diseases International, 12 (6): 645-50, 2013.

15- MORAPPANAVAR K. and GEORGE P.: Balthazar Ct Severity Index versus Apache 2 Score in Predicting the Severity of Acute Pancreatitis. Journal of Dental and Medical Sciences, 14 (8): 45-50, 2015.

16- ZHANG X.M., FENG Z.S., ZHAO Q.H., et al.: Acute interstitial edematous pancreatitis: Findings on nonenhanced MR imaging. World Journal of Gastroenterology: W.J.G., 12 (36): 5859-65, 2006.

17- KIM Y.K., KO S.W., KIM C., et al.: Effectiveness of MR imaging for diagnosing the mild forms of acute pancreatitis: Comparison with MDCT. Journal of Magnetic Resonance Imaging, 24 (6): 1342-9, 2006.

18- LI X., ZHUANG L., ZHANG X., et al.: Preliminary Study of MR Diffusion Tensor Imaging of Pancreas for the Diagnosis of Acute Pancreatitis. PLoS One, 11 (9): e0160115, 2016.

19- LANKISCH P.G., WARNECKE B., BRUNS D., et al.: The APACHE II score is unreliable to diagnose necrotizing pancreatitis on admission to hospital. Pancreas, 24 (3): 217-22, 2002. 


\section{دور التصوير بالرنين الهغناطيسى البيكري فى تقييم حالات إلتهابات البنكريناس البغينيس الحاده}

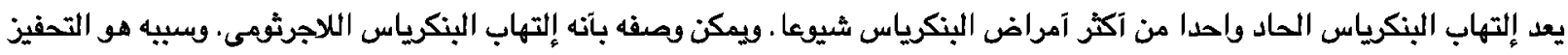

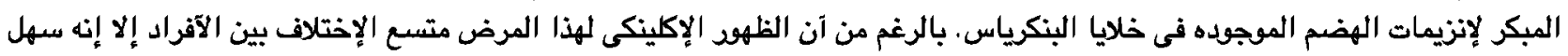

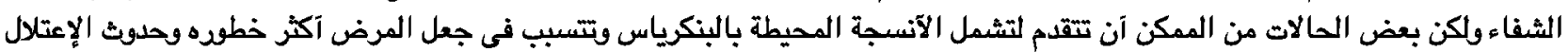
والوفاة. مقدمى الرعاية الصحية لابد وآن يتعرفوا على المرضى الآكثر عرضه للإصابة بإلتهاب البنكرياس الشعديد لكى يقدموا لهم العلاج الفعال.

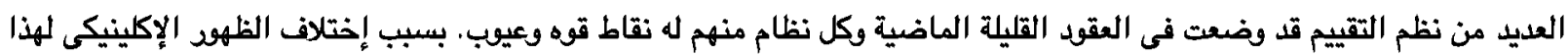

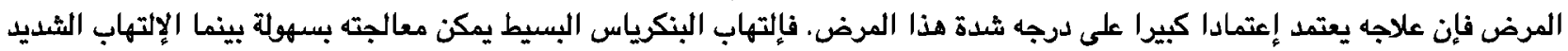

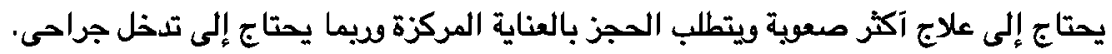
مؤشر الخطودة بالآثعة المقطعية والذى يعتمد على تقدير السوائل المحيطة بالبنكرياس ومقدار تأكل البنكرياس يعتبر المعيار الذهبى

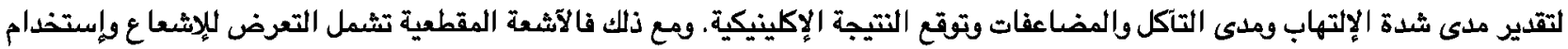

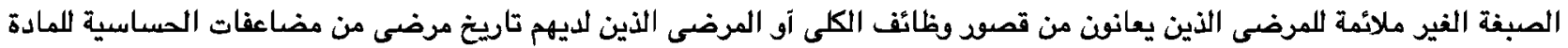
المبينة المستخدمة.

الرنين المغناطيسى يمتلك قدرة عالية في وضوح الآنسجة الرخوة وبالتالى تمييز آفضل لآنسجة البنكرياس. لذالك هدف الرسعالة هو توضيح دود التصوير بالرنين المغناطيسى في تقييم حالات إلتهاب البنكرياس الحاده.

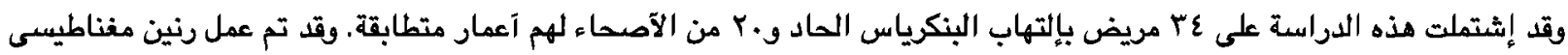
بالصبغة وتصوير بإستخدام خاصية الإنتشار ولقد تم تمليل البيانات وقد قورنت النتائج بالنظم الإكلينيكية. وقد وجد إرتباط قوى بين مؤشر الخطورة بالرنين المغناطيسى وتصنيف اتلانتا المراجع فى قياس شدة إلتهاب البنكرياس.

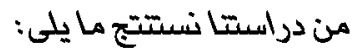
ا - التصوير بالرنين المغناطيسى بإمكانه تشخيص حالات إلتهاب البنكرياس الحاده الخفيفة وتفرقتها من باقى آسباب آلالام البطن.

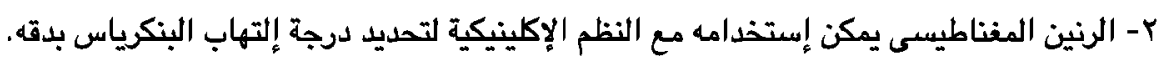

